\title{
Effect of Space Radiation on LEO Nanosatellites
}

\author{
Caleb Hillier and Vipin Balyan \\ Department of Electrical, Electronics and Computer Engineering, \\ Cape Peninsula University of Technology, Cape Town, South Africa (SA) \\ caleb.hillier@gmail.com
}

\begin{abstract}
The study is focused on the orbital mission for ZACUBE 2. The space radiations causes single bit errors. The protons and heavy ions are the main reason due to which they arises. The atmosphere for LEO is simulated on OMERE Software and was tested on TRIM in presence of different shielding effects. For all simulations involving shielding the thickness of $2 \mathrm{~mm} \mathrm{Al}$ was used. It was proven that $2 \mathrm{~mm} \mathrm{Al}$ is only effective up to $20 \mathrm{MeV}$ for protons shielding and $30 \mathrm{MeV}$ for heavy ions shielding. It is also important to note that various orbits and $\mathrm{Al}$ thickness do not affect the LET. This is important to know when trying to prevent and predict single event effects. Using TRIM the observations made in the paragraph above were re-enforced. Using this software a proton (positively charged hydrogen) was aimed and shot at $2 \mathrm{~mm}$ of aluminium. The maximum energy that the $2 \mathrm{~mm} \mathrm{Al}$ was able to stop was just over $19 \mathrm{MeV}$. As a final conclusion a $2 \mathrm{~mm} \mathrm{Al}$ shield is not able to block all the radiation that ZACUBE 2 will be exposed to, however, the majority of low energized particles will be stopped. In order to ensure that SEE does not occur additional mitigation techniques are needed to protect sensitive and vulnerable devices. These techniques could be Triple Modular Redundancy (TMR), Software EDAC schemes and others.
\end{abstract}

Key words: $\mathrm{MeV}, \mathrm{LET}$, vulnerable, techniques, sensitive, majority

\section{INTRODUCTION}

In order to understand space radiation, a study was conducted using the orbital parameters of nano satellite ZACUBE 2. The ZACUBE missions are a series of nano satellite missions being developed by FSATI in collaboration with CPUT. The aim of these missions is to develop South Africa's space industry and space experience. The first satellite of this program was launch in November of 2013 and is currently still active. The name of this initial nano satellite is the ZACUBE-1 (CPUT 2019).

The successor to ZACUBE-1 will be ZACUBE 2 (Fig. 1). The proposed launch date for ZACUBE-2 is May 2018. ZACUBE 2's primary focus is on Maritime Domain Awareness (MDA) applications (de Villiers and Van Zyl, 2015). This satellite will have the following objectives:

- Technology demonstration of AIS/VDE message reception using the primary payload

- Technology demonstration of a medium resolution imager payload

- Human Capacity Development (HCD)

- Flight heritage for F'SATI/CPUT hardware and also hardware from technology partner ESL

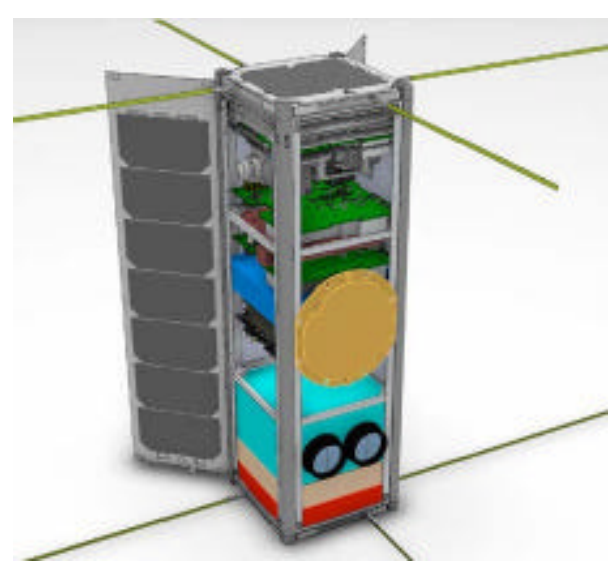

Fig. 1: ZACUBE-2 Conceptual Layout (Villiers and Van $\mathrm{Zyl}, 2015)$

These mission objectives will be achieved using the VHF AIS/VDE receiver and the Medium resolution CMOS imager acting as the primary and secondary payloads (CPUT 2016).

This will be a radiation calculation work using OMERE and TRIM Software. This resaerch will consider

Corresponding Author: Caleb Hillier, Department of Electrical, Electronics and Computer Engineering, Cape Peninsula University of Technology, Cape Town, South Africa (SA) caleb.hillier@gmail.com 
Table 1: Effects of charged particle in a space environment (Bensusen et al., 2013)

\begin{tabular}{|c|c|c|c|}
\hline Spacecraft charging & Total ionizing dose & Displacement damage & Single event effects \\
\hline $\begin{array}{l}\text { Surface charging from plasma deep dielectric } \\
\text { from high energy electrons }\end{array}$ & $\begin{array}{l}\text { Trapped protons and } \\
\text { electrons solar protons }\end{array}$ & Protons electrons & $\begin{array}{l}\text { Protons: both trapped and solar } \\
\text { Heavy Ions: both galactic cosmic rays and solar events }\end{array}$ \\
\hline Table 2: Effects of radiation on CMOS device & & & \\
\hline \multicolumn{4}{|c|}{ Space radiation environments and their effects on CMOS devices } \\
\hline \multicolumn{4}{|c|}{ Radiation source } \\
\hline Particle types & & & Primary effects in devices \\
\hline \multicolumn{4}{|l|}{ Trapped radiation belts } \\
\hline Electrons & & & Ionization damage \\
\hline Protons & & & Ionization damage; $\mathrm{SEE}$ in sensitive devices \\
\hline \multicolumn{4}{|l|}{ Galactic cosmic rays } \\
\hline High-energy charged particles & & & Single-Event Effects (SEEs) \\
\hline \multicolumn{4}{|l|}{ Solar flares } \\
\hline Electrons & & & Ionization damage \\
\hline Protons & & & Ionization damage; $\mathrm{SEE}$ in sensitive devices \\
\hline Lower energy/heavy-charged particles & & & SEE \\
\hline
\end{tabular}

the three main sources of space radiation namely: Earth Radiation Belts (ERB), Galactic Cosmic Radiation (GCR), and Solar Particle Events (SPE). This resaerch is important to prevent SEE and to assist in the correct selection of error control codes, components and shielding. Using the proposed orbital parameters set by FSATI this document will simulate and explore the environment in which the ZACUBE-2 will be orbiting.

Literature review: A sudden, usually temporary malfunction or fault of equipment is considered to be a glitch. All satellites experience glitches which can be caused by corrupted software, hardware problems, space weather, etc. The origin of a glitches and upsets be hare to determine in some case close to impossible, especially when satellites are in interplanetary space or traversing exploring unknown areas of space.

Single Events Upset (SEU) were first identified by Wallmark and Marcus (1962). SEU effect digital and electronic components, which normally result in bit flips within the memories of satellites. How SEU are cause is explained by NASA in the following manner: "when charged particles lose energy by ionizing the medium through which they pass, leaving behind a wake of electron-hole pairs"(NASA, 2012). MEU refer to errors that are induced but a single ion and cause more than one bit to flip. These upsets are normally prevented and correction using EDAC systems and hardened devices.

There are many examples of glitches and upsets which caused satellite mission to fail. The Magellan spacecraft experience both power panel and star tracker upsets after being exposed to a solar flare (Odenwald and Odenwald, 2001). Intelsat officials also that the Intelsat's Galaxy 15 telecommunications satellite has zero communication for eight months due to electrostatic discharge that caused a major software error. The source of glitches and upsets can usually be stored on board a satellite. The referred articles hold found by analysing the housekeeping data and $\operatorname{logs}$ relevance as glitches and upsets are the result of space radiation. Space radiation is a general term used to describe ionizing radiation found in space. Consisting of mainly protons and heavy ions, space radiation is made up of highly energized particles. Three mainly sources of space radiation is identified by NASA's Space Radiation Analysis Group (SRAG). "There are three naturally occurring sources of space radiation: trapped radiation, Galactic Cosmic Radiation (GCR) and Solar Particle Events (SPE) " (Langford, 2014). According to sources on radiation, SPE has the biggest impact on satellites, especially during solar maximum events. SEU and MEU mostly cause minor errors but they can in some extreme cases cause total missions to failure (Campbell, 2012). Electronic malfunctions, deterioration of materials and surface charging and discharging are all caused by radiated particles. Due to the severe impact radiation can have on a satellite, radiation is considered to be a fundamental and important factor when it comes to the design and operations of satellites (Akioka, 2009).

Table 1 summarizes the effects of charged particles in a space environment. Table 2 shows the effects of radiation on CMOS devices according to the main sources of the radiation in space. Knowing the effects of space radiation and the impact upsets and glitches can have on space missions, it is clear the EDAC systems and hardened device are need. In order to implement prevent and correction system to protect against radiation it is important to be aware of the space environment the satellite will be exposed to. Therefore, an in-depth space environment study is essential to ensure data integrity and reliability. 


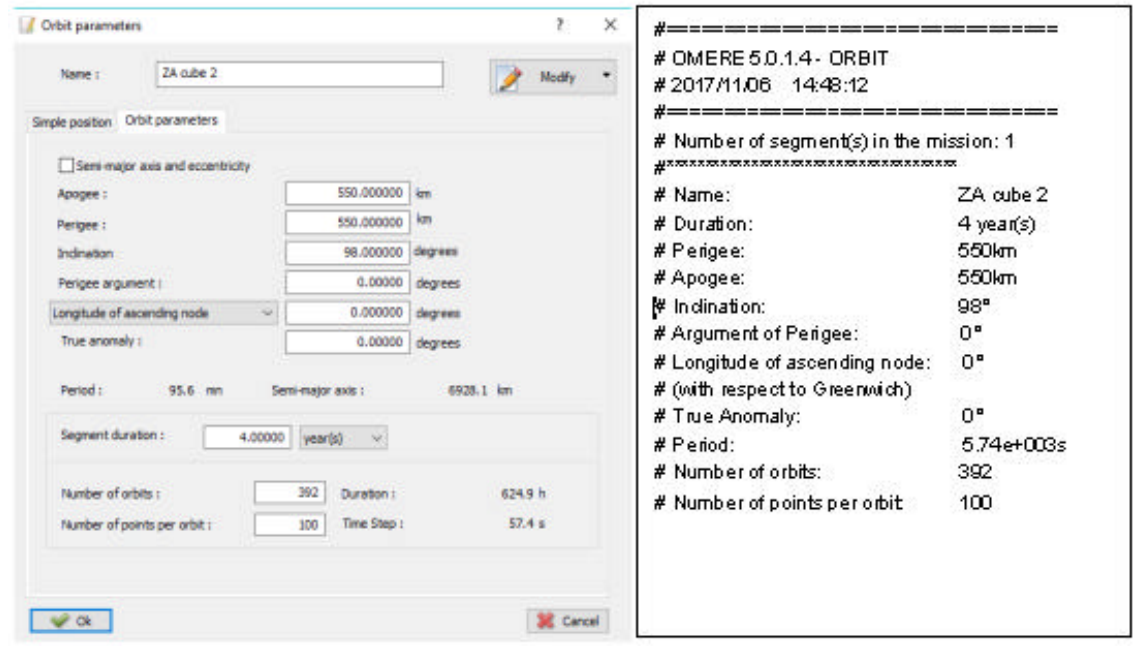

Fig. 2: a, b) Omere orbital parameter: initialisation (left) and output file (right)

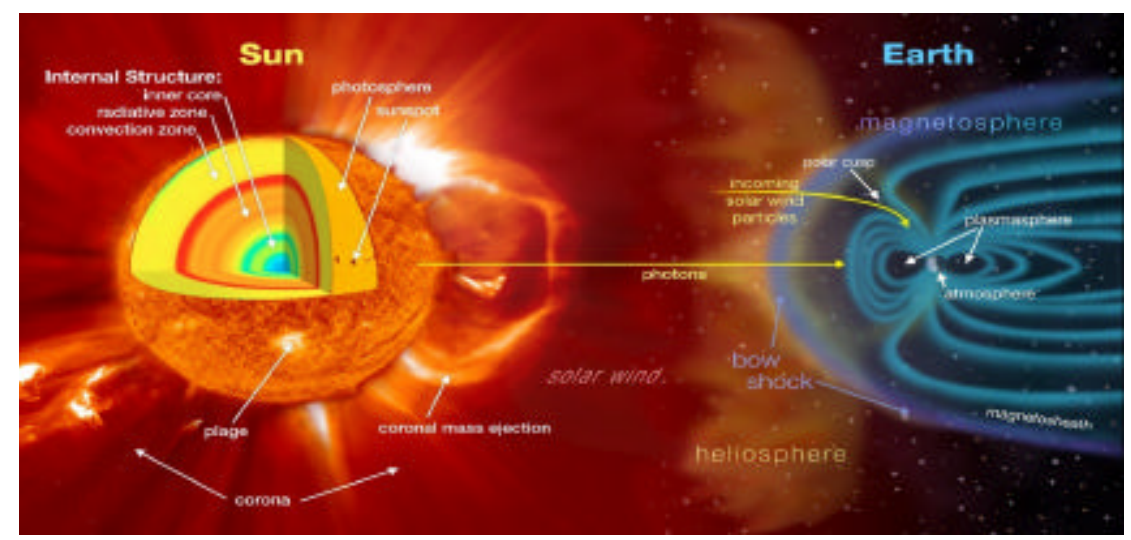

Fig. 3: Heliophysics and space weather (Bensusen, 2013)

\section{MATERIALS AND METHODS}

ZA-CUBE 2 orbital parameters: The following orbital parameters were established and entered into OMERE Software (Fig. 2) [F'SATI]. Orbital parameters are:

- Date of launch: 01/05/2018 Time: 12:00-Midday (Assumption)

- Inclination: $98^{\circ}$ (inclination for SSO orbit)

- Apogee and perigee: $550 \mathrm{~km}$ (orbital altitude under 600 $\mathrm{km}$ (Villiers and Zyl n.d.))

- Orbit type: Circular orbit

- Segment duration: 4 years (life spanned 3-5 years)

Work: With the continuous improvements and developments of technology, humans are becoming more and more dependent on technology, especially, space technology. It is a known fact that all electronic systems are affected by radiation. This could cause data to be unreliable and can result in major failures. Majorly, they are Single Event Effects (SEE).

Single event effects: SEE is electrical noise induced by the natural space environment (high energy ionising particles). This "noise" results in data corruption, transient disturbances and high current conditions which could lead to unwanted functional interrupts or in the worst case, catastrophic failures. The SEE are caused by mainly space radiation or energetic given (Fig. 3):

- Galactic cosmic rays

- Cosmic solar particles (heavily influenced by solar flares)

- Trapped protons in radiation belts 

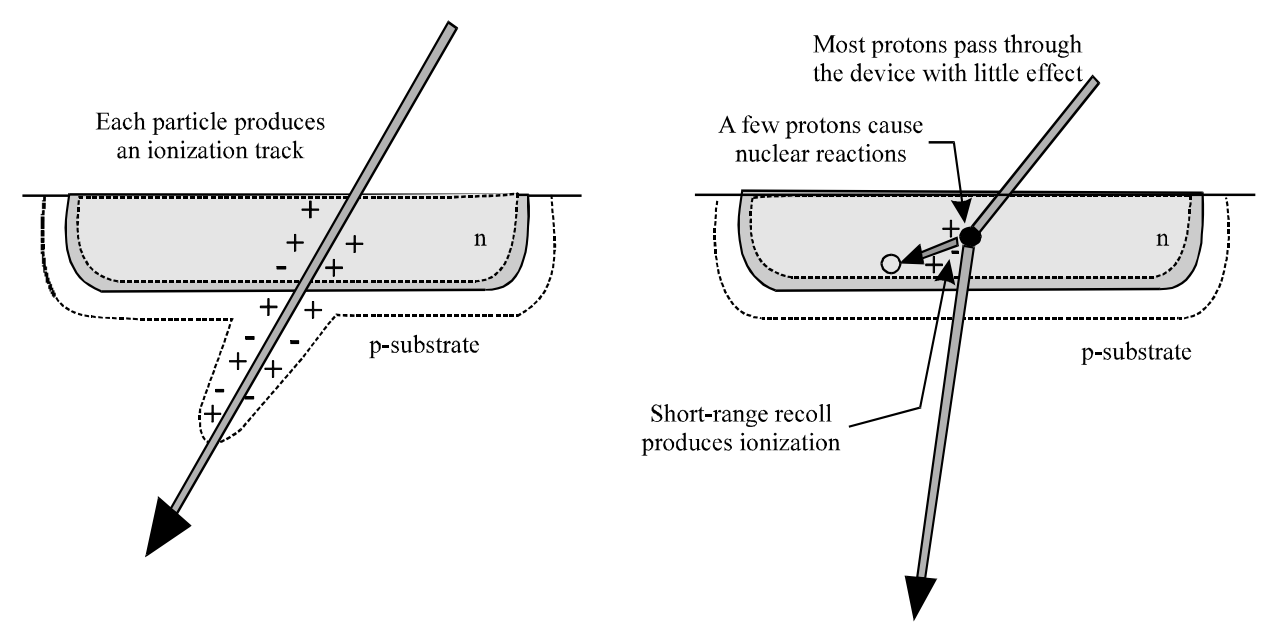

Fig. 4: Effects of heavy ions (left) and protons (right) (Halbert, 2006)

These SEE effect many types of devices and technologies. Single Event Effects includes Single Event Upset (SEU), Single Event Gate Rupture (SEGR) and others. Vulnerability to SEE has increased drastically as devices like IC's operating speeds and density increases. When radiation strikes a device, it results in the collection of charges and hence changes the electrical performance. These SEE largely impact the reliability of electronic circuits used in the space environment. The study with the help of Fig. 4 shows how particles can cause SEE within devices.

Cosmic rays: Heavy ions cause direct ionization SEE. When an ion particle travels through a device and deposits sufficient charge an event such as a memory bit flip or transient may occur. Radiation belts and solar flares.

Radiation belts and solar flares: Could cause direct ionization SEE in sensitive devices. The protons are capable of causing a nuclear reaction near a sensitive node, thus, creating an indirect ionization, effect potentially causing an SEE.

Effects of radiation in terms of SEE: SEE are usually split into two categories. These categories are listed and shown:

Non-destructive: Events which momentary or permanently change the state of a device or cell/node without affecting its functionality. For example:

- Bit flips in memory cell and registers

- Single Event Functional Interrupt (SEFI): temporal device functionality loss, recovered by a power cycle

- Single Event Transient (SET): Momentary variation in current or voltage to or from a device
Destructive: Events which interrupt device function and permanently damage the device without external interaction. For example:

- Single Event Latch-up (SEL) can cause circuit lockup, recovered by a power cycle

- Single Event Burnout (SEB) localized current in the body of a device, turning on parasitic bipolar transistor

- Single Event Gate Rupture (SEGR) dependent on the angle of incidence and on the electric field in the gate oxide

- Single Event Hard Errors (SHE) a rare case which causes a single cell unable to change state

Table 3 identifies the components and the possible effect SEE could cause.

Mitigation techniques: The mitigation techniques are methods used to reduce the severity and seriousness of SEE. There are a number of different ways to mitigate SEE.

The methods are categorised in the following levels:

- System level: TMR

- Circuit level: radiation hardened devices protecting FFs and SRAM cells

- Software level: processing+voting

- Chip-level: EDAC codes

From a mechanical point of view, it is possible to prevent SEE. This is done by using materials that have a natural resistance against radiation and by using purified fabrication materials. Large satellites make use of radiation 


\begin{tabular}{|c|c|c|c|c|c|c|c|c|c|c|c|c|c|c|}
\hline Component type & Technology & Family & Function & SEL & SESB & SEGR & SEB & SEU & $\mathrm{MCU} / \mathrm{SMU}$ & SEDR & SEHE & SEFI & SET & SED \\
\hline Transistors & Power MOS & & & & & $\mathrm{x}$ & $\mathrm{X}$ & & & & & & & \\
\hline \multirow[t]{10}{*}{ ICs } & CMOS or & Digital & SRAM & $\mathrm{X}^{*}$ & & & & $\mathrm{x}$ & $\mathrm{x}$ & & $\mathrm{X}$ & & & \\
\hline & BiCMOS SOI & & DRAM/ & $\mathrm{X}^{*}$ & $\mathrm{x}$ & & & $\mathrm{x}$ & $\mathrm{x}$ & & $\mathrm{x}$ & $\mathrm{x}$ & & \\
\hline & & & $\begin{array}{l}\text { SDRAM } \\
\text { FPGA }\end{array}$ & $X^{*}$ & & & & $\mathrm{x}$ & & $\mathrm{x}$ & & $\mathrm{x}$ & & $\mathrm{X}$ \\
\hline & & & $\begin{array}{l}\text { EEPROM/ } \\
\text { FlashEEP } \\
\text { ROM }\end{array}$ & $\mathrm{X}^{*}$ & & & & & & $\mathrm{x}$ & & $\mathrm{x}$ & & $\mathrm{X}$ \\
\hline & & & $\begin{array}{l}\mu \mathrm{P} / \mu \\
\text { controller }\end{array}$ & $\mathrm{x}$ & & & & $\mathrm{x}$ & & & $\mathrm{x}$ & $\mathrm{x}$ & & $\mathrm{x}$ \\
\hline & & Mixed & $\mathrm{ADC}$ & $\mathrm{X}^{*}$ & & & & $\mathrm{x}$ & & & $\mathrm{x}$ & $\mathrm{x}$ & $\mathrm{x}$ & $\mathrm{x}$ \\
\hline & & Signal & DAC & $X^{*}$ & & & & $\mathrm{x}$ & & & & $\mathrm{x}$ & $\mathrm{x}$ & $\mathrm{X}$ \\
\hline & & Linear & & $X^{*}$ & & & & & & $\mathrm{x}$ & & & $\mathrm{x}$ & \\
\hline & Bipolar & Digital & & & & & & $\mathrm{x}$ & & & & & $\mathrm{x}$ & \\
\hline & & Linear & & & & & & $\mathrm{x}$ & & & & & $\mathrm{x}$ & \\
\hline
\end{tabular}

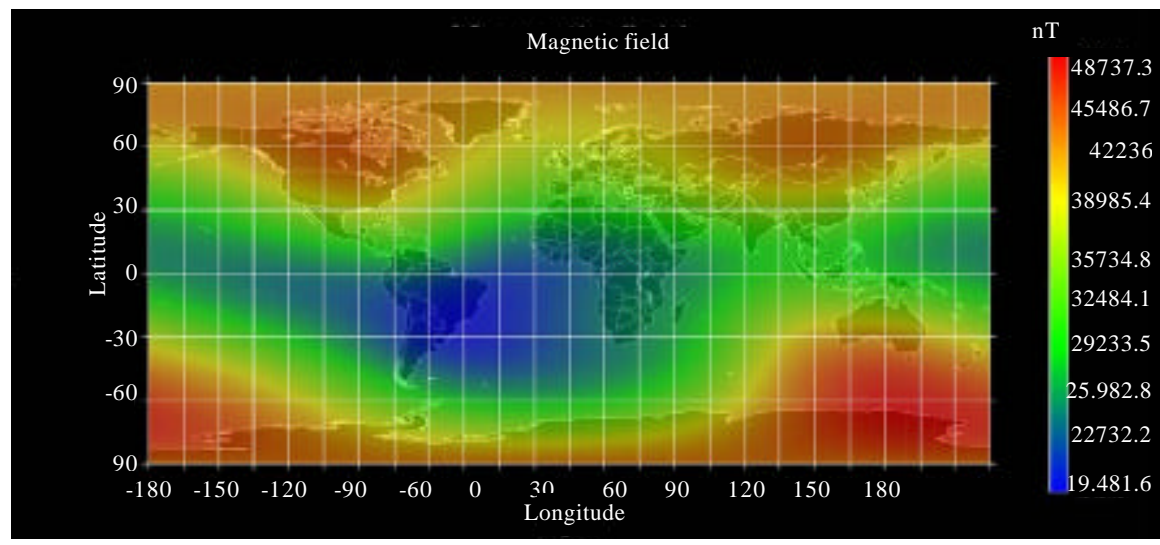

Fig. 5: Magnetic field (Jensen Cain) at $550 \mathrm{~km}$ in 2018

hardened technologies and devices which are resistant to damage and malfunctions caused by ionizing radiation. These devices and components are called RadTolerant. However, these devices are not the ideal option for nano satellites due to cost and availability in third world countries. A more practical and logical approach to prevent SEE is the satellite's PCB and component layout. For example, placing sensitive components behind the battery banks which are able to block most radiation.

Prevention is one approach to handle SEE but error detection and correction techniques can prove just as effective when implemented correctly. Redundancy on a system, software and chip level can be highly effective. Error Detection and Correction Codes (EDAC) such as Hamming and Reed Solomon (RS) codes have been used extensively in telecommunication and during deep space missions. EDAC codes are preferred as they are implemented in software and do not require too much additional power. These types of approaches are more feasible and suited for the nano-satellite industry.

Earth radiation belts: Earth Radiation Belts (ERB) are belts formed in space around Earth due to Earth's magnetic field. These belts contain and trap energised particles. The most predominant ERB is the Van Allen radiation belts.

Magnetic field: Using the flux mapping options it is possible to plot particle fluxes (AE8 and AP8 modes) and magnetic fields in a $2 \mathrm{D}$ or $3 \mathrm{D}$ views. These views and plots are effective in helping establish where magnetic fields are strongest and where protons and electrons are mostly concentrated.

The magnetic fields displayed in Fig. 5 ranges from 19.5-48.7 KnT. This plot was set up to simulate the magnetic fields at the altitude of $550 \mathrm{~km}(+/$-the altitude of ZACUBE 2) for the year 2018. The magnetic field selected for the simulation is Jensen Cain. From Fig. 5 it is clear the magnetic fields are strongest around the poles and weakest around the equator especially the South Atlantad Anomaly (SAA) area.

\section{RESULTS AND DISCUSSION}

Trapped particles: ZACUBE 2 will be traveling through the Van Allen radiation belts. For this reason, AP8 and 


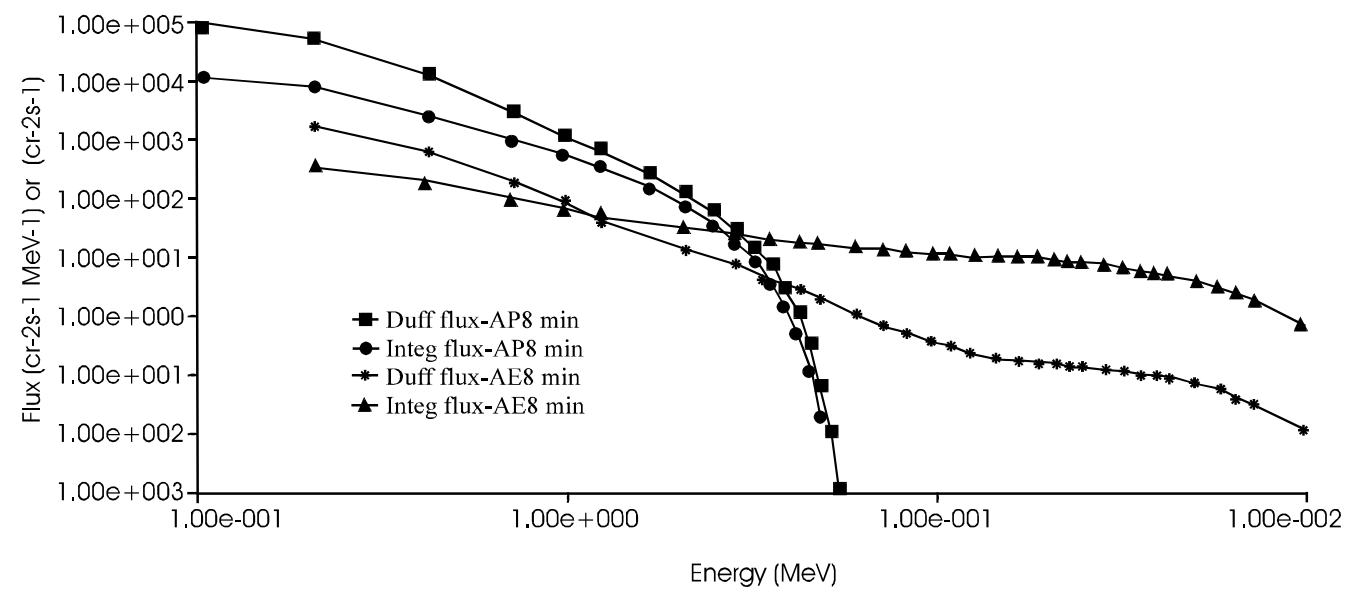

Fig. 6: Orbital average integral and differential fluxes of trapped particles

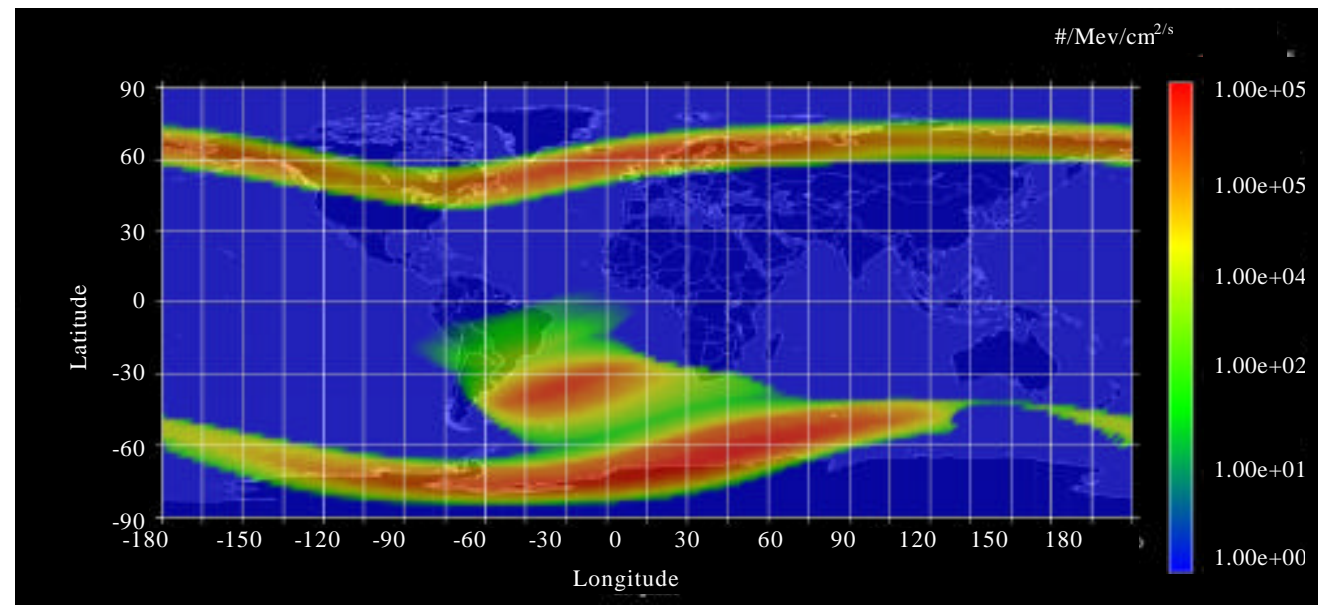

Fig. 7: Maximum trapped electrons (differential)

AE8 models were selected when simulating the trapped particles. NASA's AP8 and AE8 models have been the European standard, since, the seventies (Heynderickx, 2002) and allow for max and min models to be simulated. Mean flux was selected as an output for both the proton and electron models. The mean flux was selected as the flux spectrum is first calculated at each orbit point and then averaged.

Figure 6 shows the average integral and differential fluxes of the trapped particles within the defined orbit. From the graph, it is clear that the maximum integral flux for trapped electrons is at $8.15 \times \mathrm{e}^{4} \mathrm{~cm}^{-2} \mathrm{sec}^{-1}$ flux at an energy of a round $40 \mathrm{KeV}$, this decays to the minimum flux of $2.61 \times \mathrm{e}^{-2} \mathrm{~cm}^{-2} \mathrm{sec}^{-1}$ with a maximum of $6 \mathrm{MeV}$ energy. The maximum integral flux for trapped protons on the other hand is $1.655 \times \mathrm{e}^{3} \mathrm{~cm}^{-2} \mathrm{sec}^{-1}$ flux at an energy of around $100 \mathrm{KeV}$, this decays to the minimum flux of $1.55 \mathrm{~cm}^{-2} \mathrm{sec}^{-1}$ with a maximum of $300 \mathrm{MeV}$.
Trapped electrons: Figure 7 and 8 show the maximum trapped electrons at an altitude of $550 \mathrm{~km}$. Figure 7 (differential) and Fig. 8 (integral) shows that the trapped electrons of around $1-1 \times 10^{5} \mathrm{MeVcm}^{2} \mathrm{sec}$ for integral and differential. The trapped electrons are concentrated in a channel just below the North Pole and above the South Pole as well as the SAA Region.

Trapped protons: Figure 9 and 10 show the maximum trapped protons at an altitude of $550 \mathrm{~km}$. Figuer 9 (differential) and Fig. 10 (integral) shows that the trapped protons of around $1-1 \times 10^{5} \mathrm{MeV} \cdot \mathrm{cm}^{2} \mathrm{sec}$ for integral and differential. These protons are concentrated intensively around the SAA. This is important to note re gions of concentrated protons and electrons as they can cause SEE and in some cases to the failure of a satellite mission. 


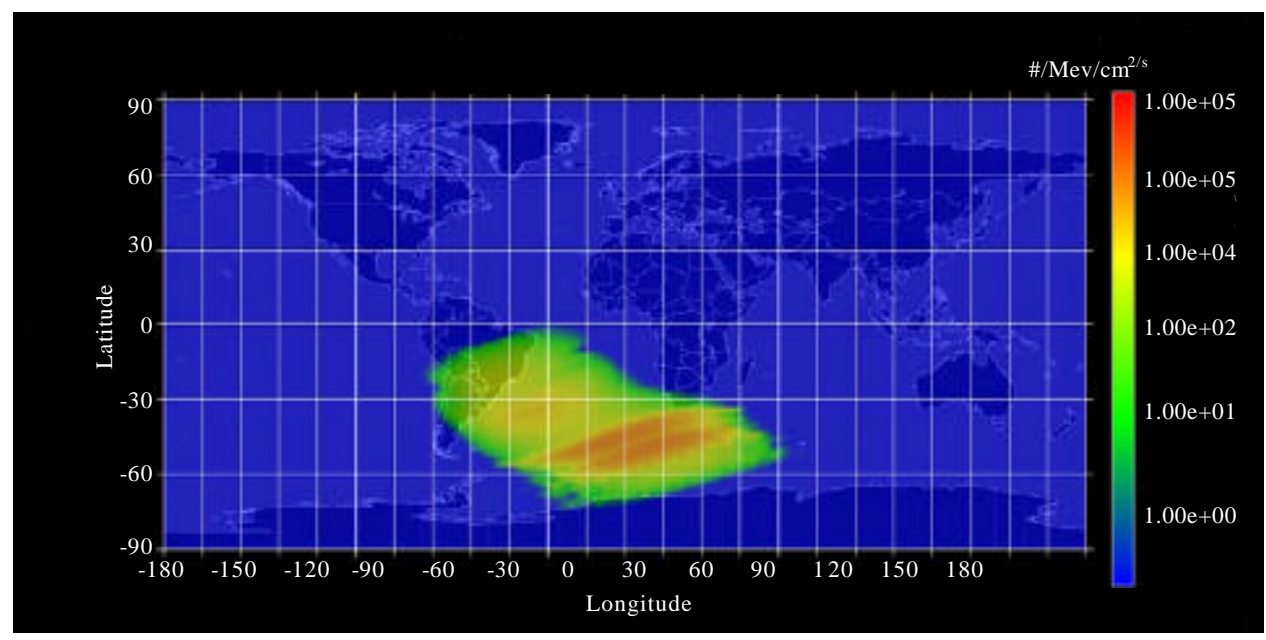

Fig. 8: Maximum trapped electrons (integral)

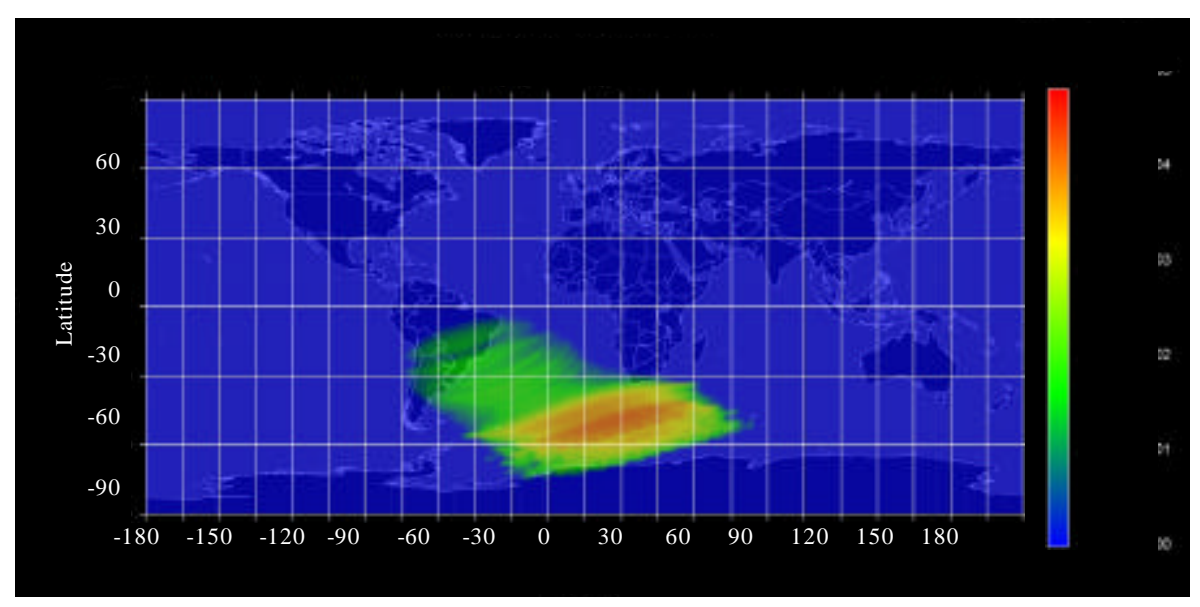

Fig. 9: Maximum trapped protons (differential)

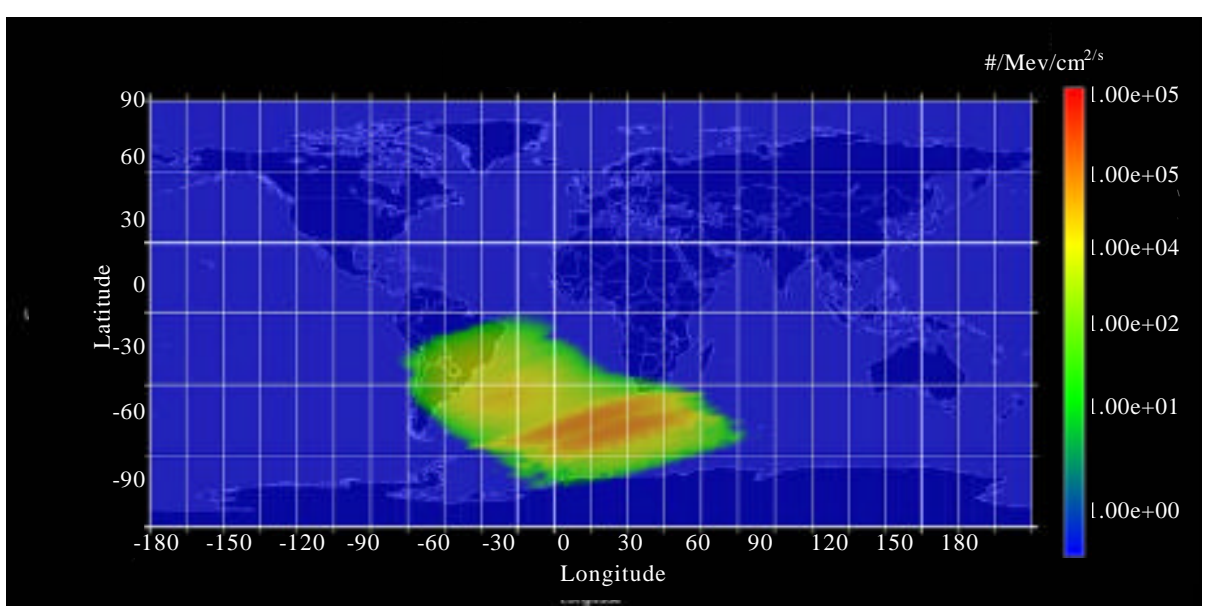

Fig. 10: Maximum trapped protons integral) 


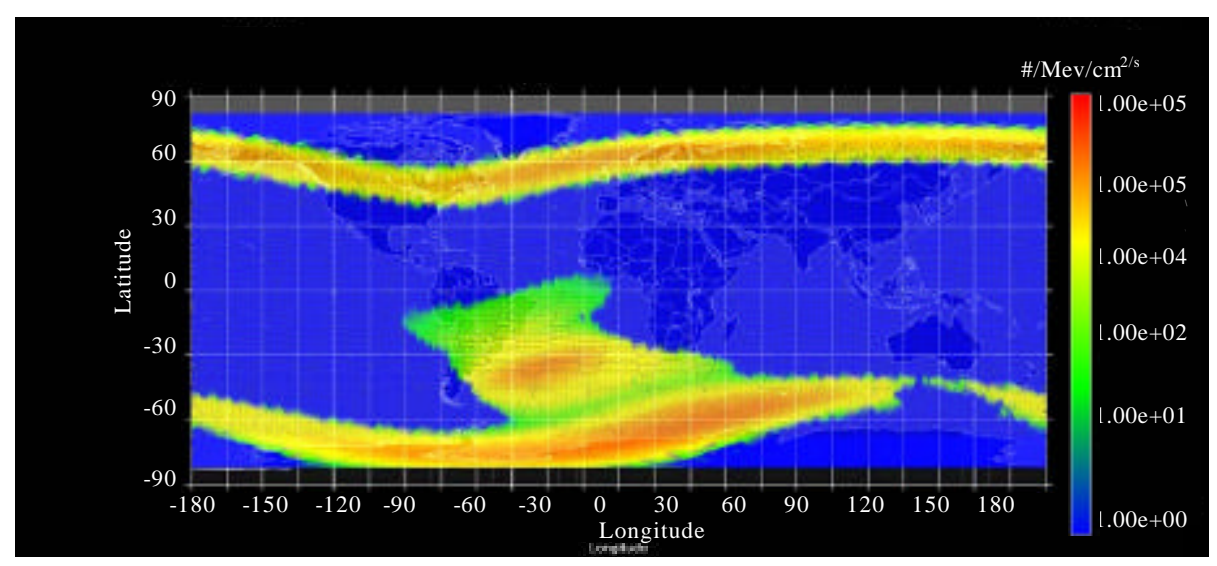

Fig. 11: Orbital minimum trapped electrons-AE8-Jensen Cain-differential

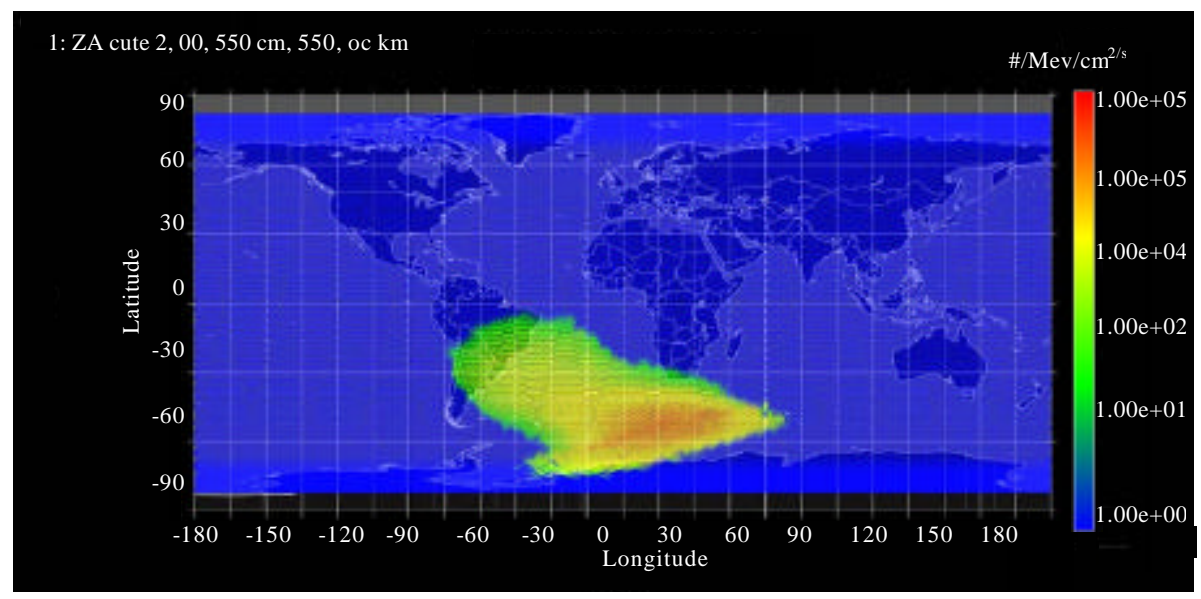

Fig. 12: Orbital minimum trapped protons-AP8-Jensen Cain-differential

Orbital trapped particles: Figure 11 show the differential intensity of the electrons and protons (Fig. 12) during a specific LEO. Their intensities reach about $10 \mathrm{MeV} \cdot \mathrm{cm}^{2}$ during the high-intensity regions such as the SAA and slight in Fig. 11 while passing the North and South Pole trapped electron channels. Satellites traveling in this orbit should be able to withst and trapped electrons of around $10 \mathrm{MeV} . \mathrm{cm}^{2}$ or new orbital parameters need to be implemented.

Solar cosmic radiation: Solar Cosmic Radiation (SCR) is one of the main sources of radiation in space. Using OMERE in Fig. 13, this radiation source is simulated on fluxes and fluences vs. energy graphs.

Protons: The proton model used is ESP, this model was selected to comply with the European Cooperation for Space Standardization (ECSS). The ECSS ensures uniformity and standardizations. For this simulation, a confidence level of $90 \%$ and a solar active period of 4 years was used. Using the plotted graph in Fig. 14, the solar protons that ZACube- 2 will be exposed to can be seen. The integral flux maximum is $1.65 \times \mathrm{e}^{2}$ protons. $\mathrm{cm}^{-2} \mathrm{sec}^{-}$ ${ }^{1}$ at an energy level of $1 \mathrm{MeV}$ and a minimum flux of $4.66 \times \mathrm{e}$ ${ }^{3}$ protons. $\mathrm{cm}^{-2} \mathrm{sec}^{-1}$ at an energy level of $3 \times \mathrm{e}^{2} \mathrm{MeV}$.

Ions: Using the OMERE Software it is possible to plot specific solar ions according to selected elements. As shown in Fig. 15 elements $Z=1$ (He/Helium) to $Z=17$ $(\mathrm{Cl} / \mathrm{Chlorine})$ are taken into account during a solar active period of 4 years. Figure 16 shows the integral and differential flux of the selected elements vs. the energy range.

Galactic cosmic radiation: Galactic Cosmic Radiation (GCR) are one of the main sources of space radiation. Using OMERE the heavy ions that ZACUBE-2 will be exposed to can be found using the generated graphs shown below. The figures show the energy verse flux graphs for galactic cosmic radiation 


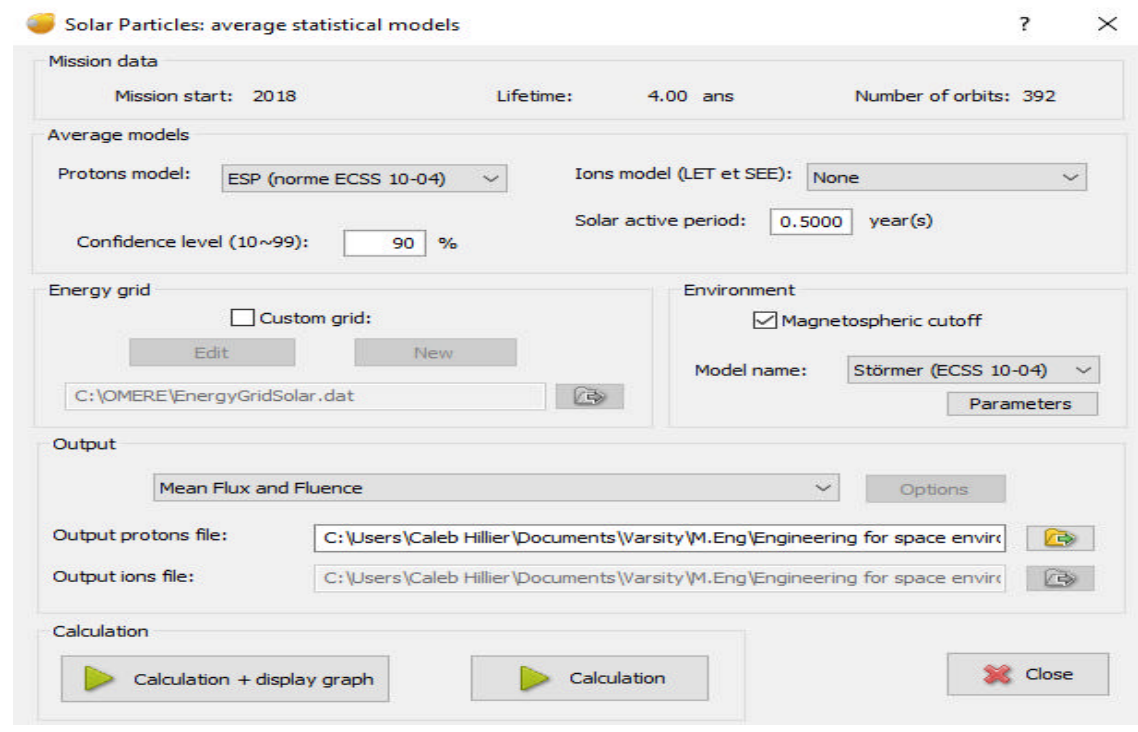

Fig. 13: Solar particle (Proton)-setupnsen cain-differential

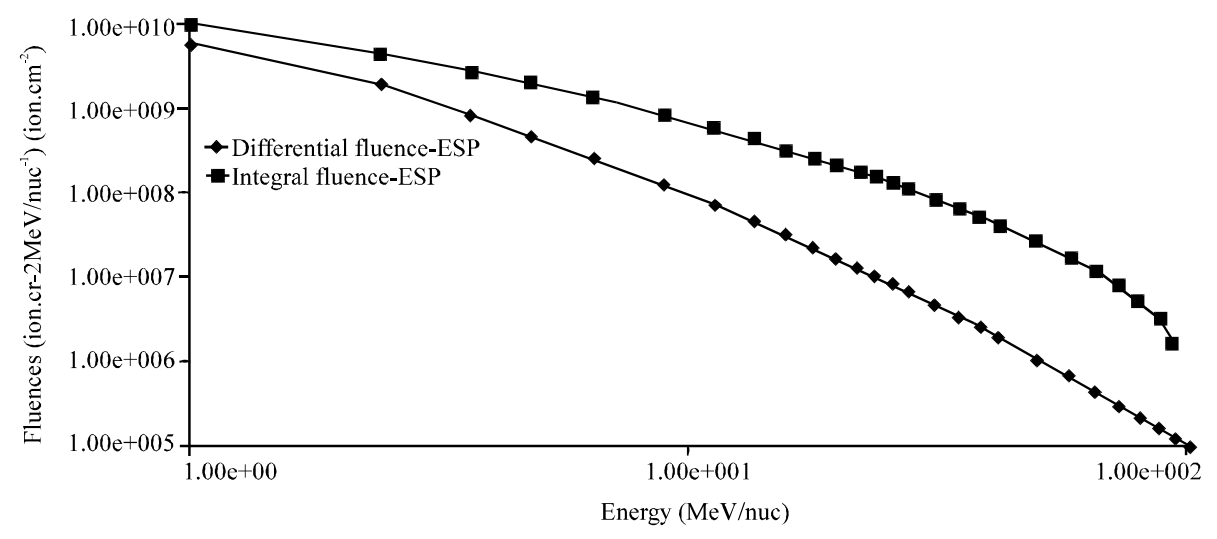

Fig. 14: Integral and differential fluences of solar protons

during a solar minimum. It can be noticed that the flux level decreases as the atomic numbers of the GCR heavy ions increase from $Z=1-Z=17$. This observation can be said for both integral (Fig. 17) and differential flux (Fig. 18). The differential flux tends to peak at $5.78 \times \mathrm{e}^{-4}$ ion. $\mathrm{cm}^{-2} \mathrm{sec}^{-1}$. $(\mathrm{MeV} / \mathrm{nuc})^{-1}$ this is a well-known feature and should be noted for shielding purposes.

Shielding: Shielding is used to protect satellites against energized protons and heavy ions in space. The type of material and coating's thickness influences how radiation tolerant a satellite is.

Dose: The dose is defined as the total amount of ionizing radiation absorbed by a material. In this case study, the ZACUBE 2 nanosatellite structure consists mainly of aluminium. Aluminium was used to reduce weight and not for its shielding properties, however, the aluminium structure does provide a minimal amount of protection.

The graph plots the different sources of radiation against the thickness of aluminium. Typically $2 \mathrm{~mm} \mathrm{Al}$ is used to shield a nanosatellite, however, this thickness should be carefully chosen by considering graphs (like below), cost and weight. From the graph, the following can be noted:

- $20 \mathrm{~mm} \mathrm{Al}$ will block majority of the radiation but will add to much weight

- $10 \mathrm{~mm} \mathrm{Al}$ will block a large portion of radiation but will still add to much weight

- $5 \mathrm{~mm}$ Al will block most of the high-level radiation but will still add to much weight 
J. Eng. Applied Sci., 14 (18): 6843-6857, 2019

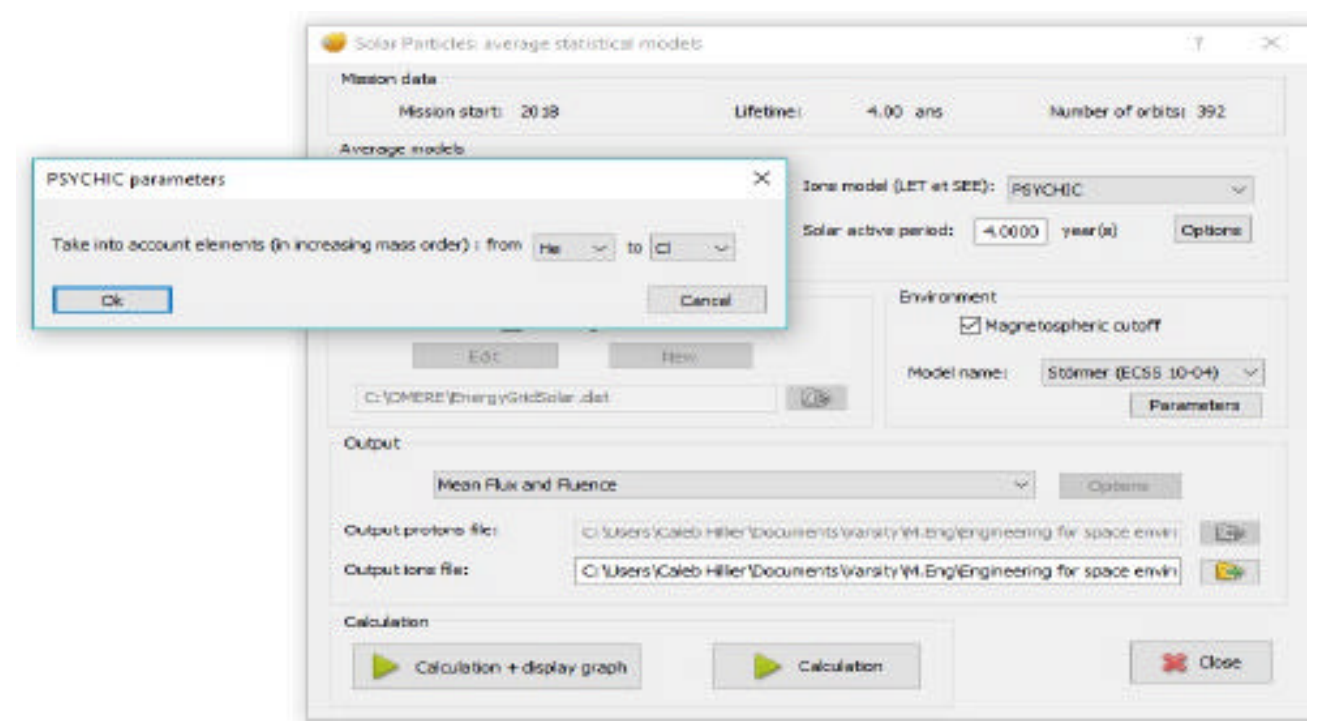

Fig. 15: Solar particle (Ion) $Z=2-Z=17$-setup

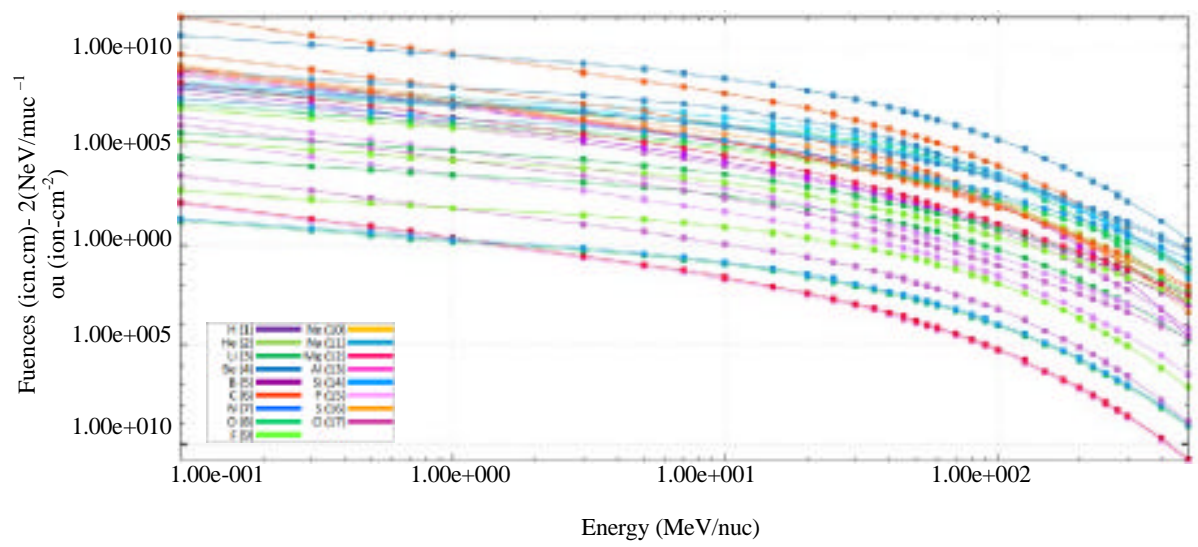

Fig. 16: Integral and differential fluence of solar ions $\mathrm{Z}=2(\mathrm{He})-\mathrm{Z}=17(\mathrm{Cl})$

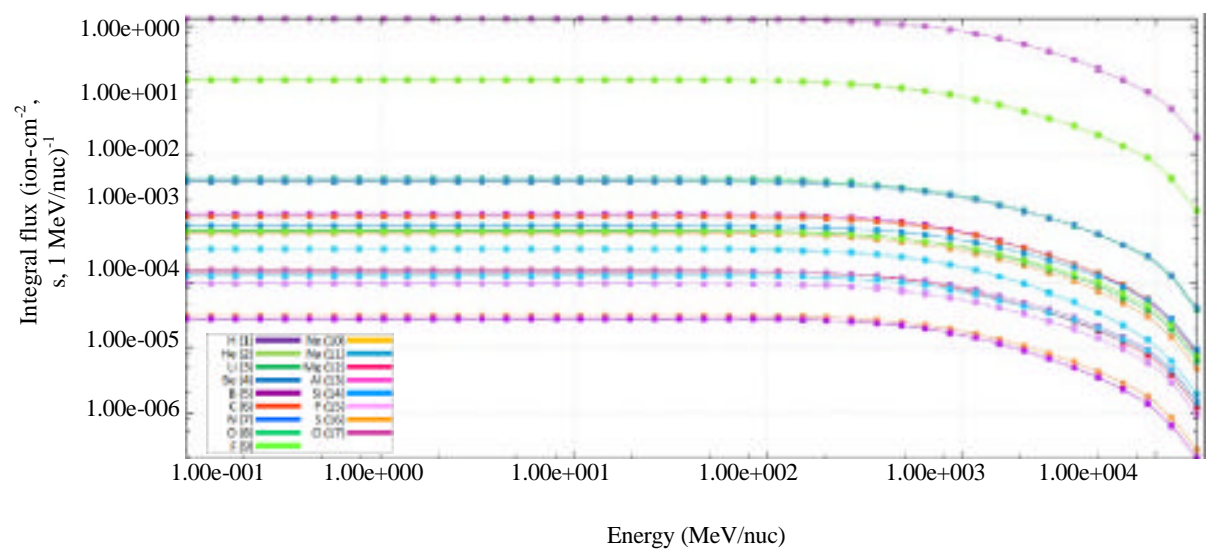

Fig. 17: Heavy ion integral $(Z=1-Z=17)$ 


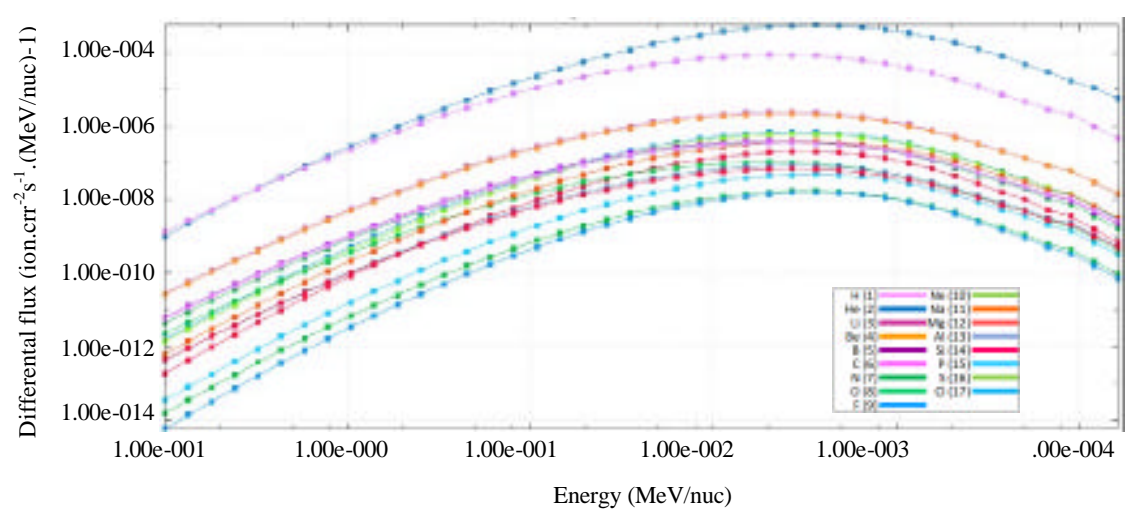

Fig. 18: Heavy ion differential $(Z=1-Z=17)$

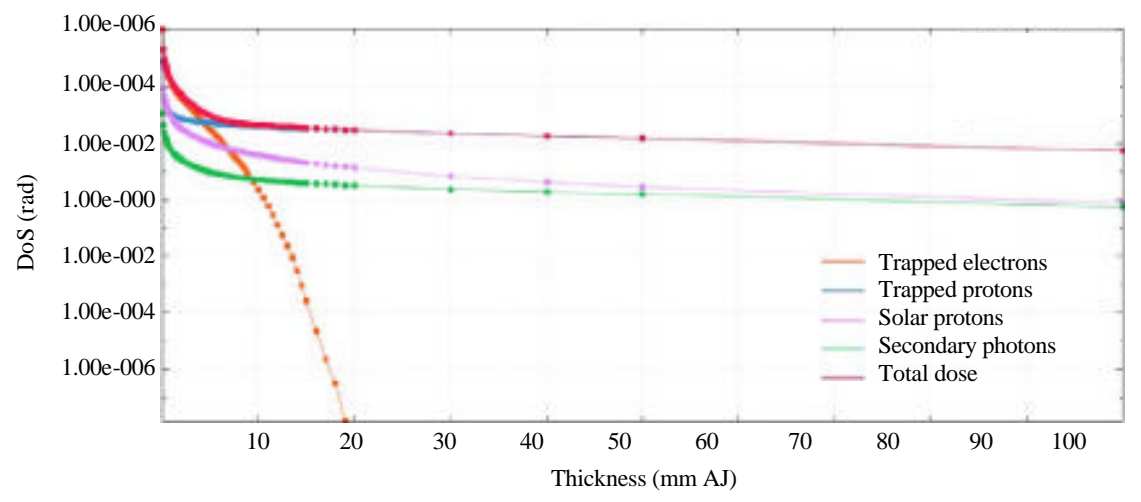

Fig. 19: Dose (rad) vs. thickness

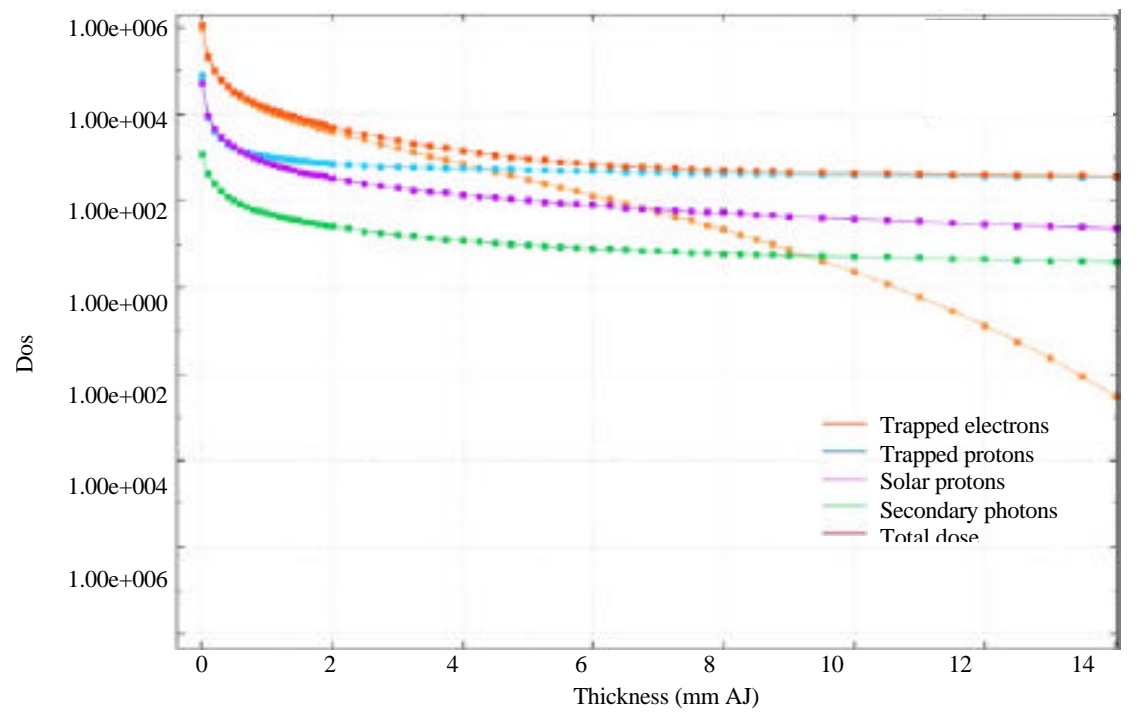

Fig. 20: Dose (rad) vs. thickness (zoom in)

The $2 \mathrm{~mm} \mathrm{Al}$ only blocks the high levels of radiation (Fig. 20). This thickness will be the best trade-off when looking at weight and cost vs. radiation. It is important to note that $2 \mathrm{~mm} \mathrm{Al}$ is used in most of the simulations as this is the standard thickness of ZA CUBE 2's casing. Aluminium is not used for its radiation prevention properties but because it is light weighted and cheap. Figure 21 shows the orbital dosage graph for a nanosatellite with a $2 \mathrm{~mm} \mathrm{Al}$ structure. Orbital rotations plotted were kept to a minimum in order to reduce the computational power needed to run the simulation. When comparing these results to the flux mapping graphs it is clear that the satellite structure will provide some 


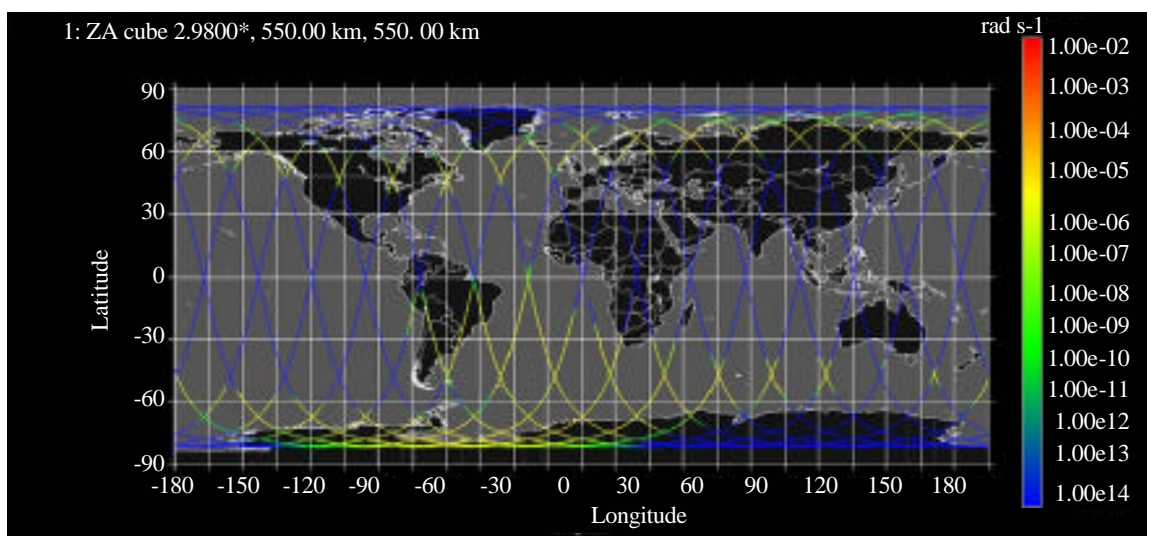

Fig. 21: Orbital dosage graph for $2 \mathrm{~mm}$ aluminium shield

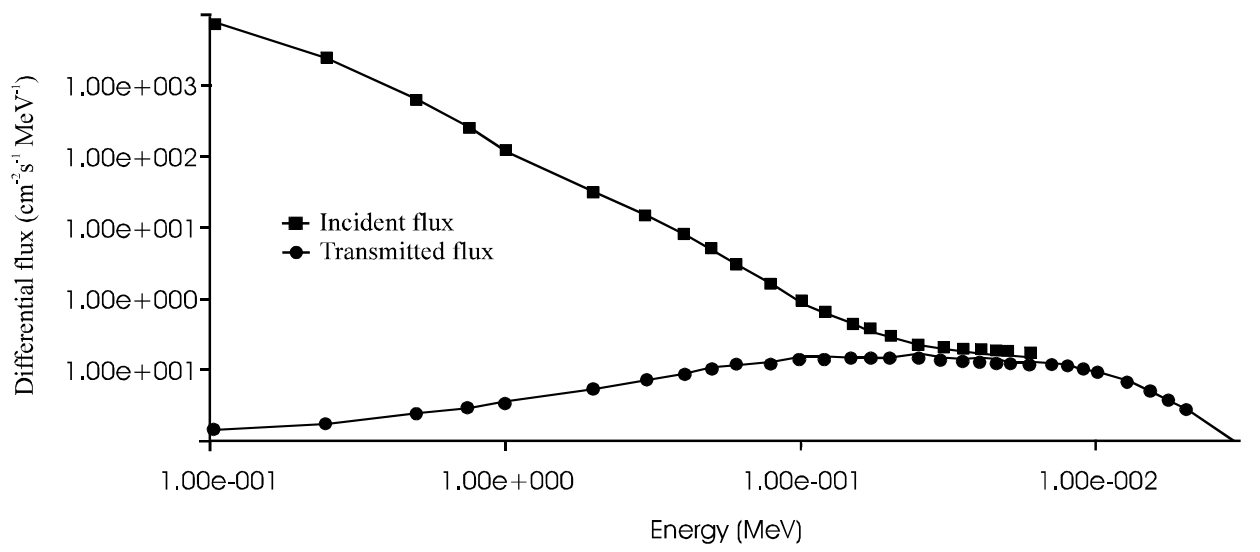

Fig. 22: Fluxes of incident and transmitted trapped protons in ERBs

shielding when traveling through the high radiation regions of space, like the SAA. From this, we can conclude that $2 \mathrm{~mm} \mathrm{Al}$ is able to provide some radiation protection but not enough.

Transport: Figure 22 and 23 below compare the incident flux and transmitted flux for trapped protons (Fig. 22) and solar peak and mean heavy ions for hydrogen (Fig. 23). The transmitted flux is the resulting flux after implementing $2 \mathrm{~mm} \mathrm{Al}$ shielding. Figure 22 shows $2 \mathrm{~mm} \mathrm{Al}$ has a very high absorption of the low energized proton but basically nothing for energies above $20 \mathrm{MeV}$. For Fig. 23, it is also

clear that $2 \mathrm{~mm} \mathrm{Al}$ shielding will help protect the satellite against heavy ions of energies $30 \mathrm{MeV}$ but is useless for energies above $30 \mathrm{MeV}$.

Linear energy transfer: Figure 24 is the calculated LET spectra graph. The graphs show the flux vs. Liner-Energy-Transfer (LET). This integral LET spectrum was plotted specifically for ZACUBE-2 orbital parameters of $98^{\circ}$ inclination with $2 \mathrm{~mm} \mathrm{Al}$ shield.
TRIM; The transport of ions into a matter: TRIM was created by James F. Ziegler and was described by him in the following manner (Ziegler, 2013). "TRIM (the Transport of Ions in Matter) is the most comprehensive program included. TRIM will accept complex targets made of compound materials with up to eight layers, each of different materials. It will calculate both the final 3D distribution of the ions and also all kinetic phenomena associated with the ion's energy loss: target damage, sputtering, ionization and phonon production. All target atom cascades in the target are followed in detail. The programs are made, so, they can be interrupted at any time and then resumed later. Plots of the calculation may be saved and displayed when needed." Using TRIM protons of different energies will be aimed at aluminium and other materials. The results will help establish how effective the material is at shielding the satellite. These results will be shown in the sections to follow.

TRIM set-up: Using the set-up tables (Fig. 25) different aspects to test can be selected. In this document the following scenario was simulated and tested: 


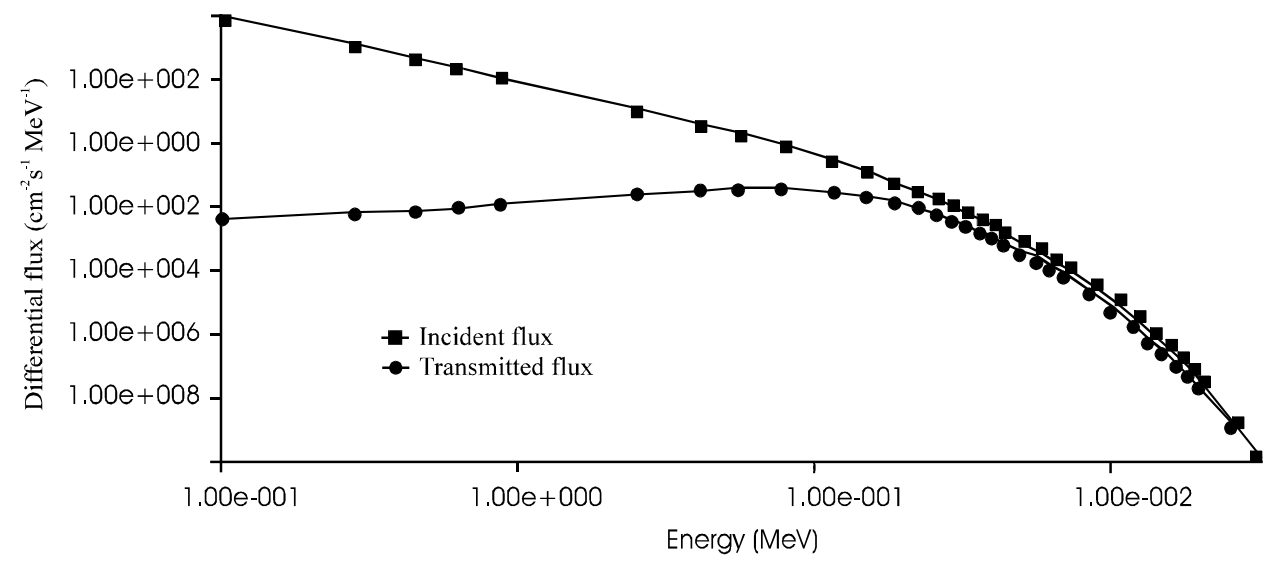

Fig. 23: Fluxes of incident and transmitted solar peak and mean heavy ions

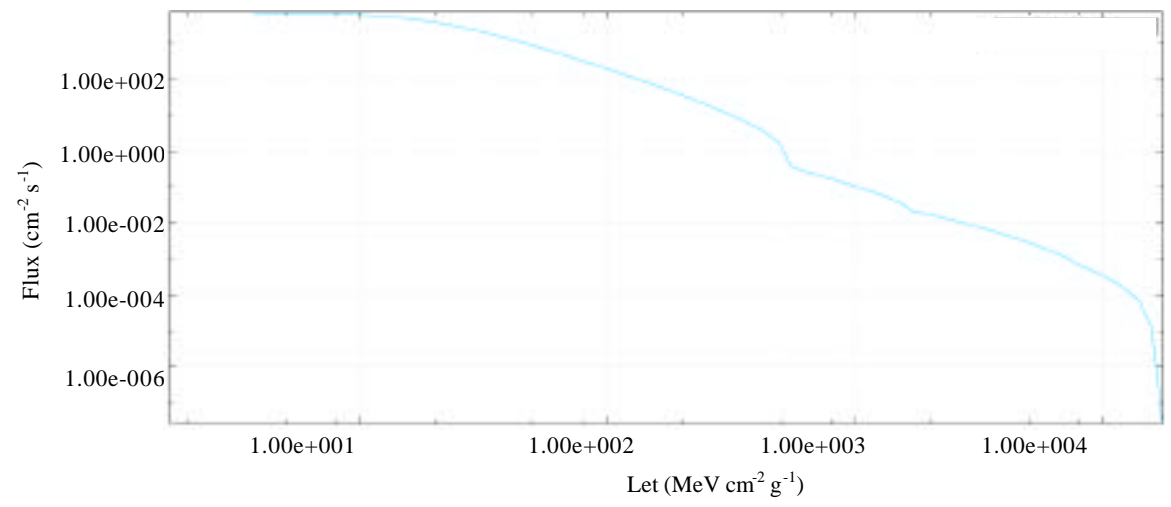

Fig. 24: Integral LET spectrum diagram for $98^{\circ}$ and $2 \mathrm{~mm} \mathrm{Al}$ shield

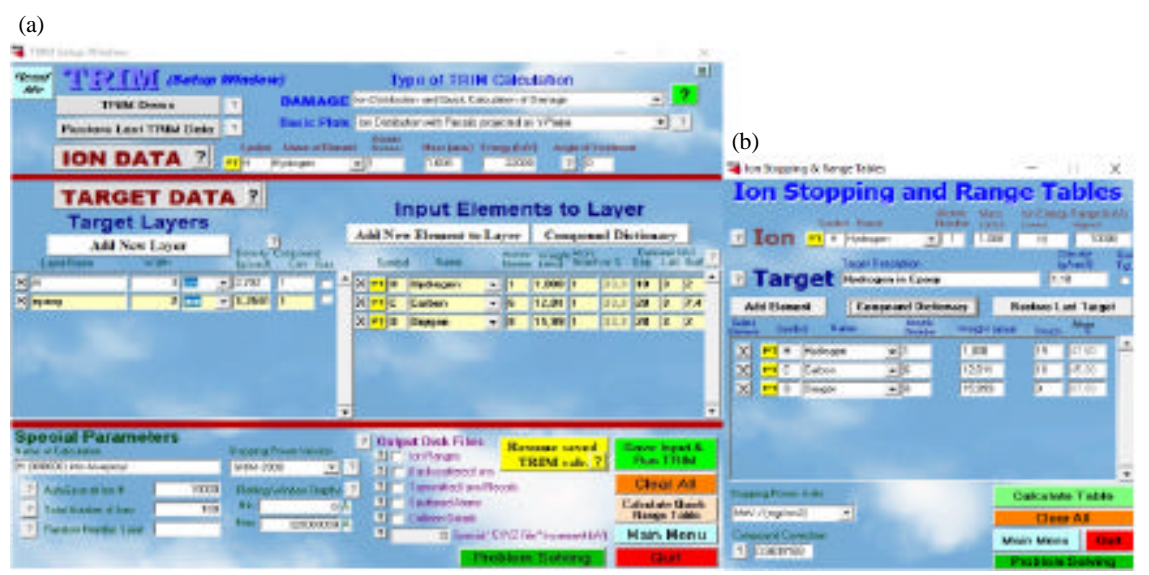

Fig. 25: a, b) TRIM set-up windows

- Protons (+Hydrogen) of $10 \mathrm{MeV}$ depth into $2 \mathrm{~mm}$ aluminium

- Protons (+Hydrogen) of $19 \mathrm{MeV}$ depth into $2 \mathrm{~mm}$ aluminium

Protons of $10 \mathrm{MeV}$ depth into aluminium: When aiming a proton of $10 \mathrm{MeV}$ at aluminium, the proton is able to penetrate to about $0.7 \mathrm{~mm}$ into the $2 \mathrm{~mm}$ Al shield. This interaction can be seen in Fig. 26.

Protons of $19 \mathrm{MeV}$ depth into aluminium: When aiming a proton of $19 \mathrm{MeV}$ at aluminium, the proton is almost able to make it through the $2 \mathrm{~mm} \mathrm{Al}$ shield. This interaction can be seen in Fig. 27. 


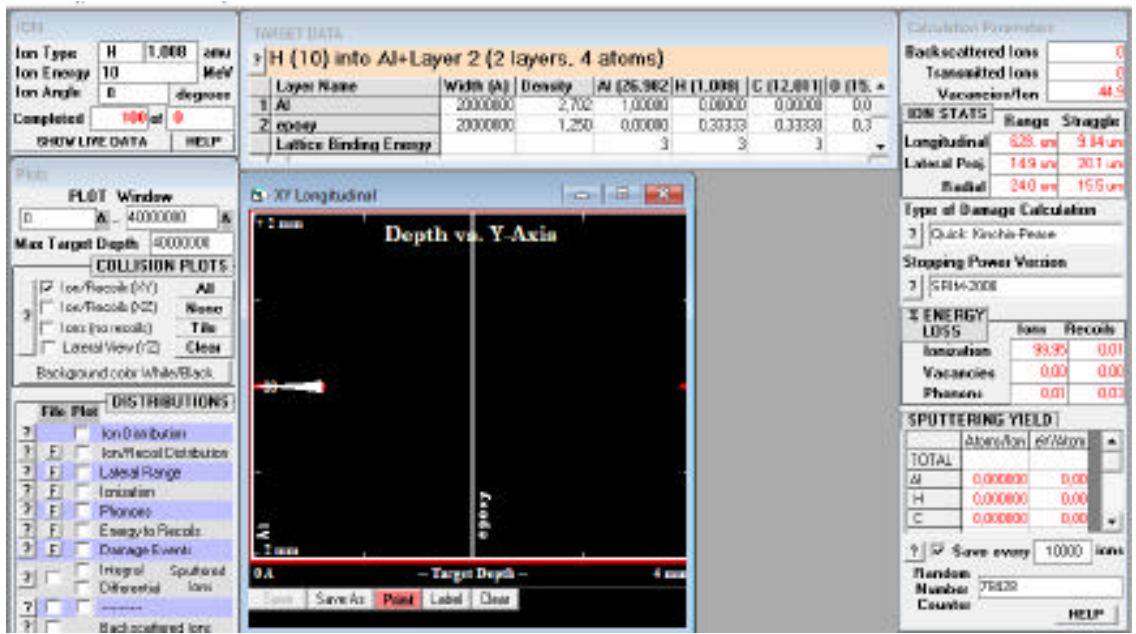

Fig. 26: Protons (+ hydrogen) of $10 \mathrm{MeV}$ depth into $2 \mathrm{~mm}$ aluminium

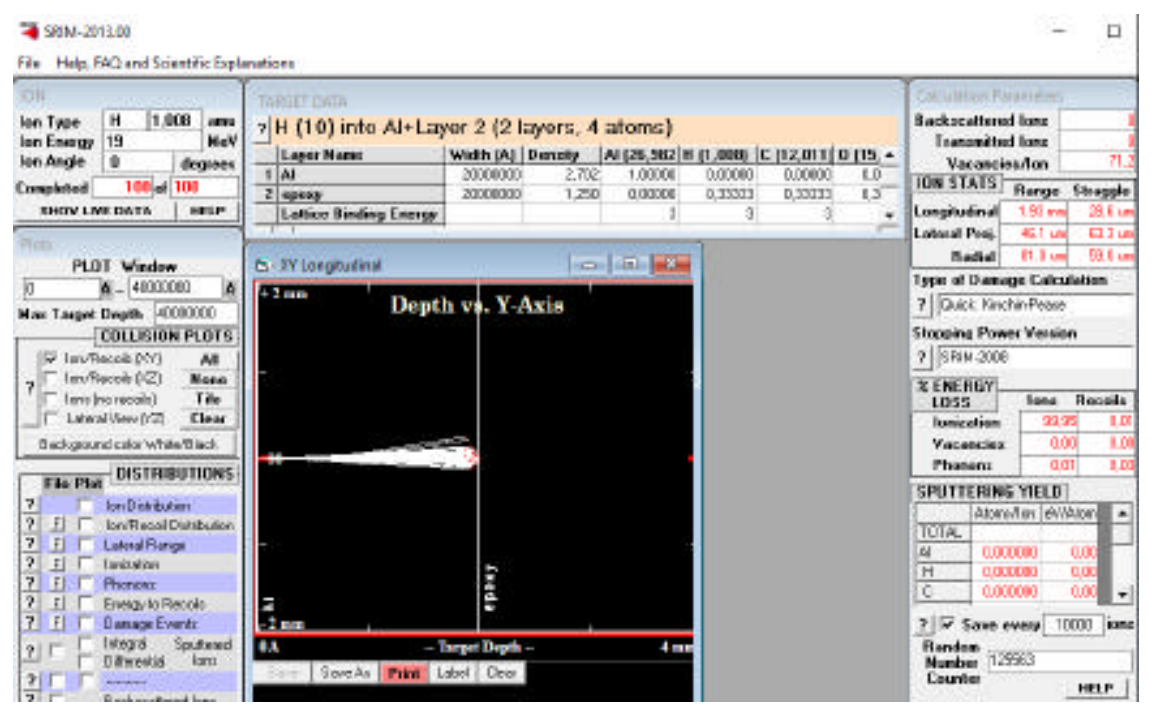

Fig. 27: Protons (+ hydrogen) of $19 \mathrm{MeV}$ depth into $2 \mathrm{~mm}$ aluminium

\section{CONCLUSION}

In this study, the orbital mission for ZACUBE 2 was simulated using OMERE Software and different shielding was tested using TRIM. From the research done during the initial stages of this study, we know that space radiation causes SEE. The main cause of these events are protons and heavy ions. There are three main sources of space radiations namely Earth Radiation Belts (ERB), Solar Cosmic Radiation (SCR) and Galactic Cosmic Radiation (GCR). Using the mentioned software, the following radiation environments that the ZACUBE 2 will be exposed to was simulated and analysed:

- Differential and integral spectra for the trapped particle in ERB
- Differential and integral spectra for the trapped particle in energetic solar particle events

- Differential and integral spectra for the trapped particle in GCR

- Transport curves for $2 \mathrm{~mm} \mathrm{Al}$ shielding at $98^{\circ}$ inclination

- LET curve for ZACUBE 2

For all simulations involving shielding the thickness of $2 \mathrm{~mm} \mathrm{Al}$ was used. It was proven that $2 \mathrm{~mm} \mathrm{Al}$ is only effective up to $20 \mathrm{MeV}$ for protons shielding and $30 \mathrm{MeV}$ for heavy ions shielding. It is also important to note that various orbits and $\mathrm{Al}$ thickness do not affect the LET. This is important to know when trying to prevent and predict single event effects. Using TRIM the observations 
made in the paragraph above were re-enforced. Using this software a proton (positively charged Hydrogen) was aimed and shot at $2 \mathrm{~mm}$ of aluminium. The maximum energy that the $2 \mathrm{~mm} \mathrm{Al}$ was able to stop was just over $19 \mathrm{MeV}$. As a final conclusion a $2 \mathrm{~mm} \mathrm{Al} \mathrm{shield} \mathrm{is} \mathrm{not}$ able to block all the radiation that ZACUBE 2 will be exposed to, however, the majority of low energized particles will be stopped. In order to ensure that SEE does not occur additional mitigation techniques are needed to protect sensitive and vulnerable devices. These techniques could be Triple Modular Redundancy (TMR), Software EDAC schemes and others.

\section{ACKNOWLEDGEMENT}

I wish to thanks the management of Cape Peninsula University of Technology, Cape Town, South Africa.

\section{REFERENCES}

Akioka, M., 2009. Space radiation effect on satellites. J. National Inst. Inf. Commun. Technol., 56: 49-55.

Bensusen, S., 2013. Heliophysics and space weather. NASA, Washington, USA. https:// svs.gsfc. nasa.gov/30481.

Campbell, K., 2012. Sumbandila provided lessons for next SA satellite. Agreenco Environmental, Southern Afric. http://www.engineeringnews.co.za/print-version/su mbandila-provided-lessons-for-next-sa-satellite-201 2-12-06

FSATI., 2017.ZZACUBE-1_French South African institute of technology. French South African Institute of Technology, South African.
FSATI., 2019. ZACube-2_French South African Institute of technology. French South African Institute of Technology, South African.

Heynderickx, D., 2002. Review on Modelling of the Radiation Belts. In: Matter, Anti-matter and Dark Matter, Battiston, R. and B. Bruna (Eds.). World Scienctific, New York, USA., ISBN:981-238-118-10, pp: 87-96.

Langford, M., 2014. Space radiation analysis group-NASA. Johnson Space Center, 2101 E NASA Pkwy, Houston, TX 77058, USA. https://srag.jsc.nasa.gov/spaceradiation/What/Wh at.cfm

NASA., 2012. NASA thesaurus. National Aeronautics and Space Administration, Washington, USA. https://www.sti.nasa.gov/nasa-thesaurus/\#.XHy2k bg6 $\mathrm{GmU}$

Odenwald, S.F. and S. Odenwald, 2001. The 23rd Cycle: Learning to Live with a Stormy Star. Columbia University Press, New York, USA., ISBN:9780231120784, Pages: 219.

Wallmark, J.T. and S.M. Marcus, 1962. Minimum size and maximum packing density of nonredundant semiconductor devices. Proc. IRE., 50: 286-298.

Zell, H., 2013. Radiation belts with satellites-NASA. NASA, Washington, USA. https://www. nasa.gov/mission_pages/sunearth/news/gallery/20 130228-radiationbelts.html

Ziegler, J., 2013. SRIM-The stopping and range of ions in matter. Srim Enterprises, LLC, New York, USA., http://www.srim.org/

de Villiers, D. and R. van Zyl, 2015. ZACube-2: The successor to Africa's first nanosatellite. J. Satellites Sci., 1: 1-5. 